\title{
Estereotipos de género transmitidos a los niños y niñas en la familia postpatriarcal
}

\section{Alicia Noemi Bonelli ${ }^{1}$ \\ Universidad Argentina de la Empresa}

\section{Ensayo}

Material original autorizado para su primera publicación en Journal de Ciencias Sociales, Revista Académica de la Facultad de Ciencias Sociales de la Universidad de Palermo. Recepción: 16-10-2018

\section{Aceptación: 10-3-2019}

Resumen: Desde los Estudios de Género los estereotipos de género son construcciones sociales. Estos se internalizan en la infancia en la socialización. Son la base para la construcción de la identidad de género. En la familia se reproducen y transforman los estereotipos y formas de poder que subyacen a las relaciones entre los géneros.

Este ensayo reflexiona acerca de en qué medida las transformaciones de los roles de género que se observan en las prácticas en el interior de las familias han modificado dichos estereotipos. Además, propone, desde una perspectiva crítica, "visibilizar" los procesos mediante los cuales se expresan, reproducen, transforman y transmiten, en la socialización primaria, las formas establecidas de "ser mujer" y de "ser varón" a los niños y niñas en las familias de hoy.

Esta reflexión se realiza a partir de los resultados de un estudio exploratorio cuyo objetivo fue conocer los estereotipos de género que padres y madres jóvenes transmiten a sus hijos e hijas en la socialización primaria. Los datos cuantitativos provienen de 200 encuestas respondidas por varones y 200 por mujeres, con preguntas de opción múltiple y escalas actitudinales; los cualitativos de 10 entrevistas en profundidad administradas a padres y 10 a madres, de 20 a 40 años, con hijos e hijas de 2 a 5 años del Área Metropolitana de la Ciudad de Buenos Aires.

Los resultados revelaron que los estereotipos se están transformando en el contexto familiar. Se observó la coexistencia de modos de subjetivación masculina y femenina tradicionales con modos instituyentes. Dicha tensión se manifestó en: distribución de tareas domésticas, crianza y atributos psicosociales. Los estereotipos de género transmitidos hoy a los hijos e

\footnotetext{
${ }^{1}$ Lic. en Psicología. Docente de Psicología Social e Investigadora en la Universidad Argentina de la Empresa. Docente de Psicología Social en la Facultad de Psicología de la Universidad de Buenos Aires. Correo electrónico: aliciabonelli@hotmail.com
} 
hijas no son tan antagónicos ni asimétricos como los de la cultura patriarcal, sino que se caracterizan por incluir algunos roles y cualidades psicosociales comunes a ambos géneros.

Palabras clave: estereotipos; género; infancia; socialización primaria.

\section{Gender stereotypes transmitted to boys and girls in the post-patriarchal family}

Abstract: From Gender Studies, gender stereotypes are social constructions. These are internalized in childhood in socialization and are the basis for the construction of gender identity. In the family, the stereotypes and forms of power that underlie gender relations are reproduced and transformed.

This essay intends to reflect on the extent to which the transformations of gender roles observed in practices within families have modified these stereotypes. It also aims, from a critical perspective, to "visibilize" the processes by which the established forms of "being a woman" and "being a man" are expressed, reproduced, transformed and transmitted in primary socialization. and girls in today's families.

This reflection is based on the results of an exploratory study whose objective was to know the gender stereotypes that young mothers and fathers transmit to their sons and daughters in primary socialization. The quantitative data come from 200 surveys answered by men and 200 by women, with multiple choice questions and attitudinal scales; the qualitative of 10 indepth interviews administered to parents and 10 to mothers, from 20 to 40 years old, with children from 2 to 5 years old from the Metropolitan Area of the City of Buenos Aires.

The results revealed that stereotypes are being transformed in the family context. The coexistence of traditional masculine and feminine subjectivation modes with instituting modes was observed. This tension manifested itself in: distribution of domestic tasks, upbringing and psychosocial attributes. The gender stereotypes transmitted today to sons and daughters today are not as antagonistic or asymmetric as those of the patriarchal culture, but are characterized by including some roles and psychosocial qualities common to both genders.

Key word: stereotypes; gender; childhood; primary socialization.

\section{Introducción}

La teoría feminista hunde sus raíces en los movimientos de liberación de las mujeres surgidos en los años sesenta a partir de la obra El Segundo sexo de Simone de Beauvoir (1969) en la cual enuncia el axioma: "mujer se hace no se nace" (Fridman, 2017).

A partir de allí comienza a delimitarse la categoría "género", la cual permitió visibilizar y desnaturalizar la lógica binaria y excluyente, que ordena la distribución del poder desigual 
entre varones y mujeres. Dicha inequidad comenzó a ser pensada como producto de normas culturales dejando por fuera el campo de la naturaleza.

Desde este paradigma, el género es definido por oposición al concepto de sexo. El género es concebido con el conjunto de significados que diferencian a varones de mujeres: activo/pasiva, proveedor/ama de casa, público/privado, racional/sensible, competitivo/compasiva. En oposición con esto, el sexo alude a los cuerpos de varones y mujeres, en tanto fijos, inmutables y naturales. De esta manera, el género es a la cultura, lo que el sexo es a la naturaleza.

Siguiendo esta línea, Rubin (1986) propone el Sistema de Sexo/Género como "el conjunto de disposiciones por el que una sociedad transforma la sexualidad biológica en productos de la actividad humana, y en el cual se satisfacen esas necesidades humanas transformadas" (Rubin, 1986, p. 97).

En la actualidad, a partir de la proliferación de la teoría Queer y los estudios culturales, las oposiciones binarias comienzan a ser criticadas, lo que trae aparejado el debate y cuestionamiento de la clásica distinción sexo/género (Butler, 2007).

Desde esta perspectiva teórica, antiesencialista y deconstructiva, la noción de género se extiende hasta abarcar la oposición misma de sexo/género, entendida como un efecto discursivo que ubica tal oposición por fuera de los límites del discurso, pues es el propio discurso el que genera la distinción discursiva/extra-discursivo. Por tanto, sexo y género no adquieren su valor oposicional por fuera de los significados culturales (Martínez, 2011).

El género es, entonces, un fenómeno cultural que se va construyendo en relación con las vivencias, cambios y transformaciones socioculturales (Butler, 2007).

Las representaciones culturales de género se expresan y manifiestan a través de estereotipos. Los estereotipos de género son "generalizaciones preconcebidas sobre los atributos asignados por la cultura a varones y mujeres" (Del Valle, 2002; Laird y Thompson, 1992).

Muchos son los estudios que ponen de manifiesto que estos estereotipos tienen una fuerte tendencia a persistir en el tiempo y que a través de diferentes generaciones suelen permanecer inalterables. Al respecto, Williams y Best (1990) afirman que los cambios no se producen de manera automática, sino que para que estos se modifiquen deben producirse transformaciones de los rasgos socioculturales que han permanecido a lo largo de la historia considerándose como naturales e inamovibles y que han pasado a conformar las identidades de género. 
Se entiende, entonces, que los estereotipos de género están relacionados con la cultura predominante, las formas de producción económica y la distribución del poder social en un espacio y un tiempo histórico (Morgade, 2012).

Es indudable que existen notorias transformaciones en las prácticas y dinámicas familiares en las últimas décadas. Por ejemplo, la incursión de la mujer en el mercado laboral, la cual la ha llevado a ganar autonomía económica y también poder de decisión en el interior de la familia y la pérdida del ejercicio del rol de proveedor económico único y jefe de familia de los varones, quienes hoy participan del mundo privado, realizando un porcentaje de las labores domésticas y de crianza. (Bonino, 2000; De Valle, 2002)

A su vez, como parte de estas transformaciones es importante considerar los cambios en el Código Civil y Comercial de la Nación (2015) en el cual los conceptos "responsabilidad parental" y "cuidado personal", implican una mayor compenetración de padres y madres, sean convivientes o no, para que ambos estén presentes en la vida de sus hijos e hijas.

Este ensayo se propone reflexionar acerca de si las transformaciones que se observan en las prácticas han modificado los estereotipos de género o aún falta un largo camino por recorrer para lograr relaciones más equitativas en el interior de la familia. Así mismo, tiene por objetivo, desde una perspectiva crítica, "visibilizar" los procesos mediante los cuales se expresan, reproducen, transforman y transmiten, en la socialización primaria, las formas establecidas de "ser mujer" y de "ser varón" a los niños y niñas en las familias de hoy.

Para tal fin se presentan y discuten los principales resultados obtenidos de un estudio exploratorio llevado a cabo en el Instituto de Investigaciones y Disciplinas Proyectuales de la Fundación Universidad Argentina de la Empresa durante el año 2017, el cual tuvo por objetivo indagar cuáles son los estereotipos de género, que los padres y madres de 20 a 40 años del Área Metropolitana de la Ciudad de Buenos Aires de nivel socioeconómico medio transmiten a sus hijos e hijas a través del proceso de socialización en la primera infancia.

Esta indagación parte de la concepción de que los modelos de masculinidad y feminidad con los que se sociabilizan los niños y las niñas varían según el contexto socio histórico, por lo que.se enfocó en explorar qué aspectos de los estereotipos tradicionales patriarcales coexisten hoy, en tensión con modos de subjetivación masculina y femenina emergentes $\mathrm{o}$ instituyentes en estas familias.

\section{Argumentación teórica}

En la familia, en tanto institución de transmisión de ideas, se procesan en forma permanente significados y valores de género (Morgade, 2012). Allí se reproducen y 
transforman los roles, estereotipos y formas de poder que subyacen a las relaciones entre varones y mujeres en la sociedad.

En relación con esto, Gimeno Collado (1999) sostiene que la estructura y procesos de la familia no son un simple calco de los estereotipos sociales, sino que esta es un agente de su propio desarrollo y de cambios.

Siguiendo la misma línea, Di Marco (2005) afirma:

En el discurso familiar típico de cada grupo, está contenido un repertorio de significados de género que abarca tanto los que se hablan como los que se callan (...) estos reflejan contradicciones y conflictos sobre el significado que se le atribuye a las relaciones de género" (Di Marco, p. 93; citado en Donini, 2005).

La familia es un subsistema dentro del sistema social y, puesto que una de sus funciones básicas es preparar a sus miembros para insertarse en la sociedad, esta ha ido adaptándose y respondiendo a los sucesivos cambios sociales, influyendo a su vez en la dirección y profundidad de estos, a través del proceso de socialización primaria (Falicov, 1991; Donini, 2005).

La socialización primaria es, entonces, una de las funciones principales de la familia junto con la de promover el desarrollo personal de los hijos e hijas, también denominado proceso de individuación.

Este último hace referencia al proceso mediante el cual se desarrolla el self, o propia identidad (Gracia Fuster, E. \& Musitu Ochoa, G. 2000). La mismidad se distingue por la capacidad del hombre, inherente al mecanismo del lenguaje, de ser objeto para sí mismo, esta capacidad resulta de asumir el rol de otros y así poder responderse a sí mismo desde la perspectiva de ellos. Esta se diferencia de los otros en una perspectiva relacional ya que se constituye por referencia a otros (Williamson y Bray, 1991).

Siguiendo a Mead, "El sí mismo no existe inicialmente, en el momento del nacimiento, sino que surge durante el proceso de la experiencia y actividades sociales" (Mead, 1963, p. 135).

Por otro lado, la socialización es el proceso a través del cual el individuo interioriza las pautas de su entorno sociocultural las cuales son mediatizadas por los agentes socializadores según sus propias interpretaciones o visiones de este.

Berger y Luckmann (1996), retomando a Mead (1963), sostienen que en este proceso los niños y las niñas se identifican con los roles y actitudes de los otros significantes y con la consideración que estos tienen de ellos, constituyendo así su identidad como una "entidad reflejada". 
Los niños y las niñas internalizan "el mundo social" de los otros significantes y con este los estereotipos masculino y femenino, los que constituyen la base de la construcción de la identidad de género (González, 1999; Lagarde, 1998).

Como ya se ha planteado previamente, el género es una construcción social que alude al conjunto de ideas, normas, instituciones y expectativas compartidas en una comunidad y cultura respecto a las características, comportamientos apropiados, derechos, posibilidades, obstáculos y potencialidades de varones y mujeres. Estas son impuestas por los sistemas políticos, económicos, culturales y sociales a través de los agentes de socialización (Correa Echeverría, 2012).

Retomado el paradigma antiesencialista y deconstructivo sostenido por Butler (1999) "El género no tiene estatuto ontológico fuera de los actos que lo constituyen. En esta lectura, el género es el efecto retroactivo de la repetición ritualizada de performances" (Duque, 2010, p.88). Entendemos por performances a los "actos del habla" y a todo un repertorio de gestos corporales que obedecen a un estilo relacionado con cada uno de los dos géneros culturales que acaban naturalizándose y produciendo la ilusión de una sustancia, de una esencia. Tales producciones genéricas y sexuales se dan en el marco de la denominada por Butler, Matriz Heterosexual (Duque, 2010).

Un ejemplo de esta matriz heterosexual está en las prácticas occidentales de crianza a partir de las cuales niños y niñas tienen un lugar y un papel predeterminado en el mundo.

Entonces, las masculinidades, al igual que las feminidades son construidas, producidas, enseñadas, aprendidas, vigiladas, clasificadas y ordenadas en las relaciones de poder de una sociedad y están marcadas por las particularidades del contexto histórico cultural donde ellas surgen. Por tanto, son una invención, una construcción sociocultural significada históricamente a partir de múltiples discursos que regulan, producen jerarquías, normalizan, transgreden y legitiman saberes (Beauvoir, 1969; Bourdieu, 1995; Butler, 2007/2010; Quaresma da Silva, 2012; Scott, 1995 citados en Quaresma da Silva y Ulloa Guerra ,2013).

Estas representaciones sociales (Moscovici, 1979; Jodelet, 1986) de los géneros se expresan y manifiestan en los estereotipos de género que son un conjunto de ideas acerca de lo que es ser varón y mujer y que simplifican la realidad, dando lugar a una diferenciación de los géneros que se basa en marcar las características de cada uno, otorgándoles una identidad en función del papel social que se supone deben cumplir.

Estos discursos, en la cultura patriarcal, legitiman desigualdades y jerarquías entre los géneros y los polariza (Burin, 1993/2012). 
Los estereotipos de género están en el imaginario social definido por Castoriadis (1988) como el conjunto de significaciones que instituyen una sociedad y otorgan sentido y orden a los individuos, grupos e instituciones que la componen, transmitiéndose de generación en generación, y normalizando cánones, valores y modelos de conducta. Tiene una doble vertiente, la instituida, que es la que mantiene unida la sociedad, y la instituyente que es la responsable de los cambios en el curso de la historia. Estas significaciones, además, constituyen las subjetividades de sus individuos, las cuales son capaces de modificarse a lo largo de las épocas (Castoriadis, 1988).

Siguiendo esta línea, Foucault (1980) sostiene que los regímenes de verdad producidos por instituciones que regulan las formas de conocimiento, como por ejemplo la medicina, la escuela, la familia, el sistema jurídico, etc., operan regulando los cuerpos a nivel macro y micro, biopoder. Por los que la realidad de los cuerpos y sus deseos reside en estar históricamente determinados a través de relaciones de poder y de prácticas de resistencia.

Con relación a los estereotipos de género tradicionales, Ortega (1998) sostiene que, el cuerpo constituye el primer referente sobre el que se articulan cualidades diferenciales otorgadas al varón y a la mujer. El cuerpo masculino es significado como fuerte, enérgico y activo, mientras que el femenino como frágil, delicado, pasivo y pendiente de la belleza física. El segundo referente son las capacidades intelectuales, a los varones se les atribuye mayor habilidad en tareas que requieren racionalidad, inteligencia y competitividad que a las mujeres. El tercer referente es la dimensión afectiva y emocional, en este aspecto se asigna a los varones la cualidad de ser poco demostrativos de los afectos mientras que, a las mujeres, ser sensibles, demostrativas y cariñosas. Finalmente se incorporan los modos de comunicación interpersonal, con respectos a lo cual se sostiene que las mujeres poseen mayor habilidad para la comunicación que los varones.

Como se ha visto, en cada referente las características de lo femenino y lo masculino son claramente diferenciadas.

En cuanto a la identidad de género, es la conciencia del "soy mujer" o "soy varón", esta se produce en una etapa relativamente temprana de la vida.

Dio Bleichmar (1991) define a la identidad de género como el esquema ideoafectivo más primitivo, consciente e inconsciente, de la pertenencia a un género y no a otro.

"La asignación de género se remonta a las propuestas identificadoras que parten de la fantasmatización de los atributos sexuales en el imaginario parental. Tal atribución es del orden de la cultura y no se halla determinada exclusivamente por la biología, sino por el conjunto de las significaciones" (Blestcher, 2017, p. 36). 
Desde el nacimiento, los niños y las niñas reciben, a través de los agentes socializadores, mensajes relativos a la adscripción "femenina" o "masculina" en función de la construcción simbólica del sexo. Esta se constituye en un "estímulo social" ya que despierta reacciones en los otros significantes que modelan la percepción que los niños y niñas tienen de sí mismos, sus comportamientos y valores (Morgade, 2012).

Por lo tanto, los niños y niñas se identificarán con los atributos psicosociales que los padres y madres les asignan partir de sus propios significados de género.

"Los niños y niñas no se identifican al objeto real, sino al proyecto y formas representacionales con los que se organiza la circulación simbólica y libidinal con adultos y adultas" (Blestcher, 2017, p.37).

En este proceso se constituye la base de la identidad de género. Esta orientará, desde etapas tempranas de la vida, tanto la representación de la realidad como las acciones, pensamientos, comportamientos de los sujetos (Quesada Jiménez, 2014).

Son muchos los autores que han puesto el énfasis en sus teorías con respecto al papel que tiene el juego en el desarrollo psicosocial de los niñas y niñas. Refiriéndose al proceso a partir del cual se constituye el sí mismo, en tanto objeto para sí, Mead consideró que este surge durante el proceso de experiencia y actividades sociales. Así, delineó dos etapas en el desarrollo del sí mismo: el juego y el deporte. En el juego los niños y niñas asumen un conjunto de roles duales, es decir, el propio y el de alguna otra persona (padre, madre, maestro, etc.). Esta actividad proporciona a los niños y niñas la oportunidad de explorar las actitudes de otros hacia él. Así al asumir el rol del otro, los niños y niñas aprenden a considerarse desde el punto de vista externo. De esta manera, en esta etapa del desarrollo, el sí mismo está constituido por la organización de las actitudes particulares que tienen otras personas con respecto a ella y entre sí, en los contextos de aquellos actos sociales que ha explorado en su juego.

En el deporte, los niños y niñas van más allá de las actitudes particulares de los otros específicos. Aquí el "otro" es una organización de las actitudes de todos los que están comprometidos en el mismo proceso o actividad (Mead,1963). Arminda Aberastury en su libro El niño y sus juegos (1998) afirma que varones y niñas juegan indistintamente, por ejemplo, a alimentar y alimentarse. Sin embargo, solo los adultos, proyectando sus prejuicios sobre la diferencia de los "sexos", rechazan algunos juegos en los varones y los permiten en las niñas o viceversa.

La reflexión de esta autora remarca como el contexto familiar refuerza la diferenciación genérica, dando actividades y ofreciendo juguetes y juegos diferentes a niños y a niñas. 
Ortiz Txabarri (2015) argumenta que, a partir de los estereotipos tradicionales, a las niñas se les destinan tareas relacionadas con el hogar, servir, atender a otros; mientras que a los niños se les reservan actividades de competencia que les permiten tener un mayor control sobre el medio externo, lo cual es una forma de ir delimitando las normas de comportamiento diferenciadas según género.

Alfonso y Aguado (2012) sostienen que dicha diferenciación genérica acarrea consecuencias a largo plazo. La niña desarrolla sentimientos de fragilidad y debilidad. Sus juegos la preparan para su futuro papel de madre y ama de casa, mientras que el niño desarrolla sentimientos de fuerza y posibilidades de explorar el mundo (Colás Bravo \& Villaciervos M. ,2007; Cuervo Martínez, 2010; Ospina Botero\& Montoya Pavas ,2015).

Con relación a esto, Caldera (1989, en Mosterio García, 2010), refiere que el tratamiento diferencial es mediado por los juguetes que se proponen a niños y niñas. Los juguetes tipificados como femeninos provocan en los padres y madres conductas de acercamiento y mayor frecuencia de verbalizaciones. En cambio los juguetes tipificados como masculinos provocan en los padres más conductas de distanciamiento.

Cabe aclarar que, si bien el proceso de construcción de las identidades de género continúa en la socialización secundaria, en el grupo de pares y en la escuela, en un proceso dinámico interactivo de internalización de pautas y valores (Donini, 2005) en la socialización primaria se constituye la base de esta.

A continuación, se presenta la metodología y principales resultados obtenidos en el citado estudio exploratorio para proseguir con su discusión tomando como marco teórico los conceptos esbozados previamente. El diseño metodológico consistió en un conjunto de técnicas provenientes de las perspectivas cuantitativa y cualitativa. Para el primer enfoque se diseñó y administró una encuesta con preguntas de opción múltiple y escalas Likert ad hoc. Para ello, se elaboró una muestra no probabilística por cuotas de un tamaño de 400 casos. Esta estuvo conformada por 200 casos de varones y 200 de mujeres, teniendo en cuenta el universo bajo estudio.

Se consideró como universo varones y mujeres de 20 a 40 años de nivel socioeconómico medio, del Área Metropolitana de la Ciudad de Buenos Aires, con hijos e hijas que se encuentren en la etapa de primera infancia, en este caso, de 2 hasta 5 años.

Para el desarrollo de la perspectiva cualitativa, se administraron 20 entrevistas en profundidad, a partir de un guion de entrevista semi-estructurado. La muestra, constituida por 10 entrevistas a varones y 10 entrevistas a mujeres, fue confeccionada teniendo en cuenta el universo citado previamente. 
Las técnicas de análisis de datos fueron complementarias e incluyeron tanto el análisis estadístico de los datos cuantitativos como el análisis de contenido de los datos cualitativos.

Dichos resultados serán presentados a partir de las dimensiones de análisis consideradas en este estudio: 1. estereotipos de género femenino y masculino asociados a los roles de padres y madres en relación con las actividades domésticas y de crianza en el ámbito familiar; 2 . estereotipos de género de los padres y madres en relación con los atributos psicosociales asignados a los varones y a las mujeres 3. características psicosociales que padres y madres atribuyen a sus hijos e hijas según sus propios significados de género por medio de los actos del habla (performances); 4. actividades lúdicas que realizan los niños y las niñas y su asociación con roles estereotipados según género.

\section{Estereotipos de género asociados a los roles de padres y madres en las} actividades domésticas y de crianza en el ámbito familiar

Los resultados revelaron que, si bien, la mayoría, el 74\%, considerando el total de la muestra entre padres y madres, sostiene, desde sus discursos, que los deberes y derechos en cuanto a la responsabilidad de tareas domésticas y de crianza de varones y mujeres son los mismos, en las prácticas no se refleja dicha simetría.

\subsection{Distribución de actividades domésticas entre géneros}

Las mujeres se encargan, de manera predominante, de las tareas domésticas. El grado de participación de los padres es menor, aunque significativo.

El $82 \%$ de las mujeres y el $62 \%$ de los varones se ocupa de decidir que se comerá en el hogar; el $88 \%$ de las mujeres y el $71 \%$ de los varones limpian los platos y la cocina. Hasta aquí las diferencias no son significativas, sin embargo, estas aumentan cuando se trata de limpiar el baño (mujeres: $81 \%$ y varones: $32 \%$ ), lavar y planchar la ropa (mujeres: $82 \%$ y varones: $38 \%$ ) y arreglar el cuarto propio y de los hijos (mujeres: $82 \%$ y varones: $49 \%$ ).

En las tareas de realizar reparaciones en el hogar (varones: $76 \%$ y mujeres: $26 \%$ ) y lavar el auto (varones: 61\% y mujeres: 16\%) las diferencias son a favor de los varones. Aunque la participación de los varones en las tareas domésticas es notoria, todavía prevalece la diferencia entre mujeres y varones en lo que refiere a la asunción de la responsabilidad de estas, es decir, mientras las mujeres se "hacen cargo", los varones "colaboran".

En las entrevistas en profundidad, la mayoría de las mujeres se refirió a la participación de los varones en las tareas domésticas como "ayuda" o "colaboración" y los 
varones como "dar una mano cuando tengo tiempo" o "colaborar o ayudar en las tareas domésticas cuando la pareja se lo pide".

Estos datos están en concordancia con lo enunciado por Wainerman (2002), quien señala que cuando se trata de la conyugalidad en el cuidado de la casa, las mujeres suelen utilizar el término ayudar o colaborar para referirse a la participación del cónyuge. Esto pone de manifiesto que aun en su imaginario social tanto hombres como mujeres conciben la responsabilidad del hombre en las tareas domésticas no como un deber sino como algo "digno de admirar", lo que pone de manifiesto la pervivencia de ciertos vestigios del padre tradicional.

\subsection{Distribución de actividades de crianza entre géneros}

En cuanto a las tareas de crianza, nuevamente son las mujeres quienes se encargan mayoritariamente de estas. Si bien los varones también las desempeñan, lo hacen en menor porcentaje.

Las mayores diferencias entre padres y madres se observaron en las siguientes actividades: asistencia a controles pediátricos (mujeres: 93\% y varones: 58\%); ayuda en tareas escolares (mujeres: $88 \%$ y padres: $48 \%$ ), preparación de vianda, almuerzo, merienda, cena para los hijos (madres: $77 \%$ y padres: $32 \%$ ).

Las diferencias no son significativas en tareas recreativas, en las cuales madres y padres indicaron realizarlas en la misma proporción. Es importante resaltar que la participación de los varones en las tareas de crianza es mayor que en las tareas domésticas, esto indica que, en este tipo de tareas, habría mayor equidad en los roles de género.

El hecho de que se detecte una participación más activa de parte del género masculino con respecto a las tareas de crianza de los hijos e hijas se relaciona con los aportes de Ramos Torres (1990) y Tobio Soler (2002) citados en Carrasco y Mina García (2005), quienes sostienen que en lo concerniente a los cambios en la distribución de las tareas domésticas, en particular en tareas asociadas al cuidado de los niños y niñas, los varones se han vuelto más participativos en la medida que el nivel socioeconómico del trabajo de ambos es más elevado y recupera un imaginario del rol de género más igualitario.

Esta perspectiva más igualitaria también se presentó en las entrevistas, ya que para referirse a la participación de los padres en las tareas de crianza las mujeres, en su mayoría, usaron el término "compartir". Sin embargo, los padres reconocieron que las madres cuentan con mayores destrezas, conocimientos y recursos en el desempeño de estas por ser "la madre" o bien que ellos cuentan con poco tiempo para dedicarse a las mismas, por lo que se definen a sí mismos como "colaboradores" en dichas tareas. 
Esto revela cierta pervivencia de la identificación de estos padres con el modo patriarcal de ser varón y con la atribución a las mujeres del rol tradicional "madre", consideración no que se vio tan evidente en las mujeres.

\section{Estereotipos de género de los padres y madres en relación con los atributos} psicosociales asignados a los varones y a las mujeres

La construcción social tradicional patriarcal de los géneros basada en la lógica binaria otorga a los varones atributos psicosociales tales como la fortaleza, actividad, la racionalidad, el ser poco demostrativos de los afectos, el estar orientados hacia el trabajo y poseer una sexualidad activa y viril. En oposición al género masculino, al género femenino se le atribuye: debilidad, pasividad, sensibilidad, sexualidad ligada al amor y vocación por la maternidad.

Para la presentación y análisis de los resultados, se consideraron como ejes de análisis las cuatro de las categorías que se desprenden de la propuesta de Ortega (1998): cuerpo, capacidades intelectuales, dimensión afectiva y emocional y comunicación interpersonal. A estas categorías se agregó una quinta: actuación en la esfera erótica.

\subsection{Significación del cuerpo masculino y femenino}

Sobre la dimensión significación del cuerpo los resultados revelaron en los padres un acuerdo del $50 \%$ con respecto a la sentencia: "Los varones son fuertes y activos", mientras que el acuerdo en las mujeres fue solo del $26 \%$.

Con respecto a la afirmación: "Las mujeres son frágiles y delicadas", el acuerdo fue del $33 \%$ en los padres y del $12 \%$ en las madres. $Y$ frente al ítem que enuncia: "Las mujeres están pendientes de la belleza física", el $44 \%$ de los padres y el $26 \%$ de las madres estuvo de acuerdo.

Por otro lado, frente a la sentencia "La mujer es considerada el sexo débil" se encontró un desacuerdo tanto en padres como en madres del 53\%, sin embargo, el grado de acuerdo si se compara con el desacuerdo no indica marcada diferencia ya que fue del $37 \%$. Esto demuestra que el atributo de la mujer "sexo débil" no se encuentra francamente cuestionado, sino que se observa aún cierta subsistencia de estereotipo tradicional en la cual el de par de opuestos fortaleza y debilidad queda asociado a los géneros masculino y femenino respectivamente.

Se observa, con respecto a la significación atribuida al cuerpo masculino y femenino, mayor pervivencia de los estereotipos tradicionales en los padres que en las madres, las cuales presentaron una visión más crítica de los mismos. 


\subsection{Capacidades intelectuales}

Sobre al eje capacidades intelectuales, las cuales son atribuidas de manera diferenciada por género desde los estereotipos tradicionales, se asigna a los varones mejor desempeño en las actividades que exigen racionalidad y competitividad.

Los resultados indicaron que solo el $10 \%$ de los padres y el $5 \%$ de las madres estuvo de acuerdo con atribuir a los varones la racionalidad como rasgo distintivo con relación a las mujeres. En lo que refiere a la competitividad el acuerdo fue del $47 \%$ en padres y del $26 \%$ en las madres.

Estos resultados no son concluyentes con respecto a que se les atribuya a los varones la cualidad de ser racionales, resolutivos y competitivos como rasgo característico, lo cual pone de manifiesto que estos rasgos del estereotipo tradicional podrían estar perdiendo vigencia.

\subsection{Dimensión afectiva y emocional}

Con respecto a esta dimensión, el $58 \%$ de padres y madres se mostraron en desacuerdo con respecto a las sentencias: "Está mal visto que los hombres lloren" y "Al hombre no le gusta manifestar sus sentimientos en público". Lo que podría interpretarse como un descenso en las creencias de la "dureza" en cuanto a la expresión de afectos asociada al género masculino.

Este dato se relaciona con lo enunciado por Bejar (1993), quien sostiene refiriéndose a las masculinidades emergentes en la familia postpatriarcal: "Ya no se trata del antagonismo y la asimetría. Se escuchan voces de hombres que demandan y ofrecen afecto y ternura" ( $p$. 50) o que expresar sentimientos y sensibilidades (Subirats,1998, 2007; Connel, 2006, 2009; Olavarría, 2012).

Frente a la sentencia:" Las mujeres son afectuosas y demostrativas" el acuerdo fue del $57 \%$ en los padres y del $29 \%$ en las madres.

Del análisis de estos datos se desprende que la demostración de afectos no se presenta como un rasgo atribuido exclusivamente a las mujeres y poco presente en el género masculino, sino que esta cualidad psicosocial es otorgada, tanto a los varones como a las mujeres, aunque a estas en mayor proporción.

\subsection{Comunicación interpersonal}


Este eje indagó si se atribuye a las mujeres, conforme el estereotipo tradicional, mayor habilidad para la comunicación interpersonal que a los varones. El acuerdo con respecto a la sentencia: "Las niñas son comunicativas y sociables" que mostraron los padres fue del $48 \%$, las madres acordaron solo en el $30 \%$.

Con relación al desacuerdo, el porcentaje de desacuerdo de los padres (20\%) fue menor al que dieron las madres (46\%). En total, considerando padres y madres, el acuerdo fue del $39 \%$ y el desacuerdo del $33 \%$.

En síntesis, el rasgo comunicación y sociabilidad no pareciera presentarse como exclusivo de las mujeres.

\subsection{Actuación en la esfera erótica}

Con respecto a la manera particular de actuar en la esfera erótica de los varones, los resultados de la encuesta arrojaron un acuerdo del 34\% frente a la sentencia que enuncia que los hombres siempre piensan en el sexo y un desacuerdo del $50 \%$.

Estos datos indican que, si bien la mayoría se mostró en desacuerdo con atribuir a los varones el rasgo de ser activos y viriles en la esfera erótica, lo cual marcaría una visión menos estereotípica de los varones, el grado de acuerdo indica, aún, cierta persistencia de representaciones sociales sobre el varón que se anclan en modos patriarcales de significación de su identidad en coexistencia con la atribución no tradicional.

En cuanto a la actuación del género femenino en la esfera erótica, los resultados relevaron un alto porcentaje de desacuerdo (84\%) frente a la afirmación "Las mujeres solo tienen sexo cuando están enamoradas" frente a un acuerdo del $7 \%$.

Los datos precedentes marcan cierta tendencia a atribuir a las mujeres rasgos no coincidentes con el estereotipo tradicional en el cual las mujeres se hallan representadas por medio de la dupla sexo/amor.

Esta tendencia puede pensarse como consecuencia de uno de los cambios o liberaciones que viene produciéndose en torno a la construcción de identidad femenina denominada "autonomía erótica" entendida como el divorcio entre sexo, amor y maternidad. Esto se vincula con la desnaturalización de la histórica ecuación mujer=madre y con la consiguiente valoración y legitimación de otros deseos por fuera del deseo de maternidad en las mujeres (Rosenberg, 2017).

Esta tendencia también se observó en las respuestas de los encuestados frente a la sentencia "Las mujeres no son infieles si están enamoradas". En este caso, el porcentaje de acuerdo fue del $27 \%$ (mujeres: $32 \%$ y varones: $12 \%$ ), siendo el grado de desacuerdo del 
57\% (varones: $62 \%$ y mujeres :51\%). Estos resultados muestran una mirada crítica con respecto al estereotipo tradicional, aunque el grado de acuerdo revela, al mismo tiempo, cierta permanencia del estereotipo femenino tradicional que liga a las mujeres a la dupla amor/fidelidad.

Cabe resaltar que el porcentaje de acuerdo fue más elevado en las mujeres que en los varones, lo que indicaría que la identidad de estas se encuentra, aún y en cierta manera, anclada en modos patriarcales de ser mujer.

Estos resultados están en sintonía por lo expresado por Rosenberg (2017), quien sostiene que la identidad de las mujeres en la estructura patriarcal se constituye alrededor de las relaciones amorosas. Esta tendencia se ha denominado como el "ser para otros", mientras que los varones estructuran su subjetividad en función de las relaciones de poder y de la construcción de prestigio, lo que ha sido representado por la noción de "ser para sí".

Esto significa que, desde la lógica capitalista, los fundamentos de la subjetividad de los varones se hallan ligados al poder económico, mientras que, en las mujeres, en el poder del amor, principalmente dentro de la familia. Por otro lado, esta autora plantea que, aunque las mujeres se mueven más libremente en lo que hace a su sexualidad, en comparación con lo que ocurría en otros momentos históricos, hoy en día la posibilidad de elección de compañero amoroso está más restringida si se compara con la situación de los varones.

Así mismo, según refiere Meler (2017), pese a que aún las modalidades amatorias reflejan desigualdad entre los géneros, están surgiendo "nuevas mujeres" que optan por rescindir el "contrato sexual" (Pateman, 1995), destinando la investidura amorosa a disfrutar de su propia autonomía, metas personales y hacia otros vínculos familiares y amistosos, entre otros.

Estos aportes explican, tal como se observó en los resultados, la coexistencia en tensión de modos de "ser mujer" tradicionales con instituyentes.

3. Características psicosociales que padres y madres atribuyen a sus hijos e hijas según sus propios significados de género por medio de los "actos del habla"

El análisis de los rasgos atribuidos de modo prevaleciente por parte de los padres y madres a sus propios hijos e hijas mostró concordancia con respecto a los resultados presentados hasta el momento.

Los atributos psicosociales que los padres y madres otorgaron a sus hijos en mayor porcentaje fueron: inteligente, activo, demostrativo, compañero y sociable. 
En el caso de las niñas, las cualidades que padres y madres les asignaron en mayor proporción fueron: inteligente, demostrativa, sensible, activa y sociable.

El análisis comparativo entre los rasgos atribuidos a niños y niñas por parte de padres y madres arrojó los siguientes resultados: el ser activo/a fue atribuido por el $53 \%$ de padres y madres a los niños, y por el 35\% a las niñas; la sensibilidad, por el $43 \%$ de padres y madres a las niñas y por el $25 \%$ a los niños; la fortaleza fue un rasgo adjudicado a los niños por el $18 \%$ y a las niñas por el $4 \%$ de la muestra. El ser demostrativo/a fue un adjetivo utilizado para caracterizar a las niñas por el $51 \%$ de los padres y madres y por el $43 \%$ para los niños.

Estos datos revelan cierta pervivencia de los estereotipos de género tradicionales en lo que respecta a la concepción del cuerpo masculino en cuanto que prevalece la atribución de actividad y fortaleza para los niños y en lo que respecta a la dimensión afectiva y emocional en las mujeres, dado que los padres y madres asignan a las niñas la cualidad de la sensibilidad de manera preponderante.

Sin embargo, pese a esta pervivencia en algunos aspectos de los estereotipos tradicionales, también se observó que el atributo "típicamente femenino" de ser demostrativo, también fue asignado a los niños, aunque que en menor porcentaje que a las niñas. Lo mismo ocurre con el atributo, "típicamente masculino", activa, el cual es también asignado a las niñas, aunque en menor proporción que a los niños. Estas atribuciones "instituyentes" fueron realizadas predominantemente por las madres.

Otros atributos psicosociales como: inteligente; sociable; comunicativo/a; compañero/a y competitivo/a no fueron asignados de manera diferencial para caracterizar a los niños y a las niñas.

Los resultados presentados muestran diferencia con la investigación de Maccoby realizada en 1980 (en Mosterio García, 2010) quien observó que los padres y madres describían, desde estereotipos antagónicos, a los hijos como activos, fuertes e inteligentes; y a las niñas como cariñosas, sensibles, sociables.

Aquí se identificaron cualidades atribuidas comunes a ambos géneros, lo cual podría dar cuenta de ciertas transformaciones en los estereotipos tradicionales coexistiendo con las modalidades instituidas de masculinidad y feminidad.

Dichas transformaciones pueden ser pensadas como consecuencias de los cambios en las prácticas. Esto significa que, dado que los varones ya no tienen reservado el poder económico y con este los atributos que legitiman dicho poder como la inteligencia, la actividad y la competitividad ya que lo comparten con las mujeres que también participan de él, estas cualidades ya no son asignadas exclusivamente al género masculino, sino que también comienzan a ser atribuidas a las mujeres. Lo mismo puede pensarse con respecto al poder 
del amor y la maternidad, el cual tampoco es hoy un poder exclusivo de las mujeres, puesto que los varones han comenzado a participar en las tareas de crianza. Por lo tanto, los atributos como la demostración de afecto y la sensibilidad han dejado de ser asignados de modo exclusivo a las mujeres, ya que los varones también empiezan a ser vistos con cualidades para desempeñarse en dichas tareas.

Estos cambios, si bien coexisten con cierta pervivencia de los modos tradicionales de masculinidad y feminidad, dan cuenta de la incidencia de las prácticas en la modificación de los estereotipos de género.

\section{Actividades lúdicas que realizan los niños y las niñas y su asociación con} roles estereotipados según género

Con respecto a los juguetes utilizados por niños y niñas los resultados reflejan que aún prevalece la división de estos por género, por lo que la tendencia antes mencionada no se manifiesta de manera tan marcada en este ámbito.

Es decir, los juguetes que utilizan los niños, en su mayoría, son juguetes asignados tradicionalmente a los varones.

Según refieren los padres y madres de la muestra, el $77 \%$ de los niños juega con pelota de futbol; el $68 \%$ con autos; el $62 \%$ con muñecos de superhéroes; el $48 \%$ con robots y dinosaurios. Lo mismo ocurre con los juguetes con los que juegan las niñas; los resultados indican que el $84 \%$ de las niñas juega con muñecas; el $61 \%$ con princesas; el $60 \%$ con cosméticos; el $56 \%$ con cocinitas y el $41 \%$ con juegos de té.

Se observa entonces, considerando los juguetes que fueron mencionados en mayor proporción, la persistencia del uso de juguetes según género conforme a los estereotipos tradicionales. A saber, para los varones juguetes que proponen el uso de la fuerza, la actividad y el dominio y para las niñas juguetes en los cuales se incentiva el desempeño de actividades vinculadas a las tareas domésticas, el cuidado de la belleza física y la maternidad.

Esta misma tendencia está presente en los juegos que realizan niños y niñas, los cuales, al igual que los juguetes, siguen estando diferenciados por género.

Según refieren los padres y madres, el $58 \%$ de los niños juega a la lucha; el $51 \%$ a disfrazarse de superhéroe; el 37\% con juegos virtuales como GTA y Minecraft, mientras que el $77 \%$ de las niñas realiza juegos de roles como mamá, maestra, etc.; el 62\% a disfrazarse de princesa y el $52 \%$ al karaoke e imitación de artista. 
En el uso de juguetes y juegos neutros o no asignados a un género en particular como, por ejemplo: bicicleta, juegos de mesa y masas, escondida y juegos de computadora no se encontraron diferencias en su uso entre niños y niñas.

Cabe resaltar que, aunque en menor el porcentaje, en comparación con el porcentaje de niños y niñas que usan juguetes y realizan juegos tradicionales según género, hay niños que usan juguetes y realizan juegos tradicionalmente asignados a las niñas y niñas que usan juguetes y realizan juegos tradicionalmente asignados a varones.

En el caso de los niños, el $9 \%$ juega con cocinitas, el $8 \%$ con juegos de té, $5 \%$ con muñecas, el $4 \%$ con cosméticos y el $2 \%$ con princesas.

En el caso de las niñas, los porcentajes aumentan: el $28 \%$ juega con pelotas de fútbol, el $21 \%$ con muñecos de superhéroes, el $15 \%$ con autitos, el $13 \%$ con dinosaurios y robots y el $14 \%$ juega a la lucha con los padres.

Estos datos ponen de manifiesto que, si bien los juguetes siguen estando diferenciados por género, también, aunque en menor medida, hay padres y madres que ofrecen a sus hijos/as un universo de juguetes más diverso, sin divisiones entre juguetes para niños y para niñas.

Algunos padres argumentaron que, si un varón juega con "cosas de nena, esto podría causarle trastornos en su sexualidad (homosexualidad)", aunque no son tan críticos con respecto al hecho de que una niña juegue con juguetes de varón como, por ejemplo, con una pelota, lo cual revela cierta pervivencia del estereotipo tradicional masculino, el cual tiene como rasgo característico la "homofobia".

Una minoría de las madres y padres entrevistados dijeron que proponen a sus hijos e hijas un universo diverso de juegos y juguetes, los dejan elegir en la juguetería según sus propios gustos sin dividirlos por género, lo cual pone de manifiesto cierta crítica a los estereotipos tradicionales.

Estos padres y madres también indicaron que suelen ser criticados por personas de su entorno por sus modos de proceder en torno a la decisión de los juegos y juguetes, lo cual les produce cierto malestar e incomodidad al sentirse cuestionados y culpabilizados por sus decisiones.

\section{Conclusión}

El modelo de familia patriarcal tradicional comenzó a experimentar ciertos cambios a raíz de los movimientos feministas de los años 30 y la incursión de la mujer en el mercado laboral, lo cual condujo a iniciar un proceso de transformación en lo que respecta a la 
concepción antagónica y asimétrica de los géneros y llevó al cuestionamiento de un orden natural que incluía una rígida división del trabajo entre varones y mujeres.

Fernández $(1993,2001)$ sostiene que las categorías mismas de lo femenino y lo masculino han entrado en revisión, generando una crisis en el conjunto de relaciones entre hombres y mujeres, como también las relaciones de las mujeres consigo mismas y de los hombres consigo mismos.

En sintonía con Fernández, Flaquer (1998) argumenta que estos cambios instauraron un modelo de familia postpatriarcal, el cual tiene como rasgo distintivo el papel menguante que desempeña el padre en cuanto a ocupar el lugar de proveedor único y la independencia de unos con otros sin permitir el renunciamiento de sus derechos como personas.

A partir de lo antedicho, se sostiene que la familia está siendo partícipe de los procesos permanentes de producción de lo nuevo y de conservación de lo tradicional (Freixas, 2000).

Se concluye, a partir de los resultados obtenidos en este estudio, los cuales se refieren exclusivamente a la población tomada como muestra pero que podrían estar poniendo de manifiesto una cierta tendencia en las familias de este tipo, que aunque todavía existe cierta pervivencia de los estereotipos de género tradicionales en la desigualdad en la distribución de tareas domésticas y de crianza, esta coexiste con movimientos instituyentes los cuales redefinen las relaciones entre los roles de género de manera menos asimétrica. Esto se observó principalmente en las tareas de crianza en las cuales los varones no son solo "colaboradores", como ocurre con las tareas domésticas, sino que las "comparten" con las mujeres.

Esta tensión entre lo tradicional y lo nuevo también está presente en la significación del cuerpo masculino concebido aún como fuerte y activo, en la atribución hecha a las mujeres acerca de su carácter sensible y en la diferenciación por género de los juegos y juguetes que aún es mayoritaria.

Sin embargo, también se encontró que atributos típicamente femeninos como la demostración de afecto, habilidad para comunicarse y la sociabilidad también son asignados a los varones Asimismo, atributos típicamente masculinos como la inteligencia y la actividad, también son asignados a las mujeres y que algunos padres y madres son críticos con respecto al uso de juegos y juguetes asignados por género.

Las citadas transformaciones dan cuenta de que las prácticas están modificando los estereotipos de género, el tradicional antagonismo entre la división del poder entre varones y mujeres, económico y del amor, respectivamente está perdiendo vigencia, lo cual trae aparejados cambios en la manera como de concebir la masculinidad y feminidad 
Por lo tanto, los modelos de masculinidad y feminidad transmitidos a los niños y niñas no presentan el mismo antagonismo que los estereotipos tradicionales, sino que estos constituyen modelos de identificación en los cuales coexisten en tensión algunos aspectos de los modelos tradicionales con otros aspectos instituyentes que proponen una concepción más igualitaria de los géneros.

Esta tendencia a la disolución de la polaridad entre los géneros podría tener impacto en los niños y niñas de hoy, ya que podrían estar internalizando una visión de los géneros y de sí mismos, en tanto varón o mujer, desde una perspectiva no basada exclusivamente en la oposición sino más igualitaria, es decir, con algunas cualidades y atributos comunes entre géneros.

Cabe resaltar que las mujeres mostraron una mayor tendencia a desnaturalizar los estereotipos tradicionales que los varones. Este dato podría ser un indicativo de una diferencia, entre varones y mujeres, con respecto al grado de distanciamiento o ruptura de los estereotipos tradicionales.

Estas tensiones visibilizan el proceso de transformación de los estereotipos y prácticas, aunque aún falta recorrer un largo camino para consolidar a una concepción equitativa de los modos de ser varón y mujer.

\section{Referencias bibliográficas}

Aberastury, A. (1998). El niño y sus juegos. Buenos Aires: Paidós

Alfonso, P. \& Aguado, J.P. (2012). Estereotipos y Coeducación. El Bierzo: Consejo Comarcal del Bierzo.

Béjar, H. (1993). La cultura del yo. Madrid: Alianza Editorial.

Berger, P. y Luckmann, T. (1996). La construcción social de la realidad. Buenos Aires: Amorrortu.

Blestcher, F. (2017). Infancias trans y destinos de la diferencia sexual: nuevos existenciarios, renovadas teorías. En I. Meler (Comp), Psicoanálisis y género: escritos sobre el amor, el trabajo, la sexualidad y la violencia (pp. 21-48). Buenos Aires: Paidós.

Bonino, L. (2000). Los varones hacia la paridad en lo doméstico, discursos sociales y prácticas masculinas. En C. Sánchez-Palencia y J.C. Hidalgo, (Eds.), Masculino plural: Construcciones de la masculinidad (pp. 23-46). Lleida: Edicions de la Universitat de Lleida.

Burin, M. (1993/2012). Masculinidades y Femineidades: Identidades laborales en crisis. En C. Hazaki (Comp.), Crisis del patriarcado (pp. 63-78). Buenos Aires: Topia 
Butler, J. (2007). El género en disputa. El feminismo y la subversión de la identidad. Barcelona: Paidós.

Carrasco, M. J. y García Mina, A. (2005). El ajuste trabajo familia desde una perspectiva de género. Madrid: Universidad Pontificia Comillaz.

Castoriadis, C. (1988). Los dominios del Hombre. Encrucijadas del laberinto. Barcelona: Gedisa

Colás Bravo, P. \& Villaciervos Moreno, P. (2007). La Interiorización de los Estereotipos de Género en Jóvenes y Adolescentes. Revista de Investigación Educativa, 25(1), 35-58.

Connell, R. W. (2006). Desarrollo, globalización y masculinidades en Careaga, Gloria y Salvador Cruz. Debate sobre masculinidades. Poder, desarrollo, políticas públicas y ciudadanía, (pp. 185-210). OUEG / UNAM. México.

Connell, R.W. (2009). Dentro de la Torre de cristal: La construcción de las masculinidades en el mundo del capital financiero. En J. Olavarría (Ed.), Masculinidades y globalización. Trabajo y vida privada, familias y sexualidades (pp. 15-35). Red de Masculinidades: UAHC.

Correa Echeverría, C. (2012). Abc de género. Buenos Aires: Acercándonos.

Cuervo Martínez, Á. (2010). Pautas de crianza y desarrollo socioafectivo en la infancia. Perspectivas. Psicológicas, 6(1),111-121.

De Beauvoir, S. (1969). El segundo sexo. Buenos Aires: Siglo Veinte.

Del Valle, T. (Coord.) (2002). Modelos emergentes en los sistemas y relaciones de género. Madrid: Narcea.

Dio Bleichmar, E. (1991). El feminismo espontáneo de la histeria. Madrid: Siglo XXI.

Donini, A.; Faur, E.; García Rojas, A. y Villa, A. (2005) Sexualidad y familia: Crisis y desafíos frente al siglo XXI. Buenos Aires: Novedades Educativas

Duque, C. (2010). Judith Butler y la teoría de la performatividad del género. Revista de Educación y Pensamiento, (17), 85-95.

Falicov, C. (1991). Transiciones de la familia. Buenos Aires: Amorrortu.

Fernández, A. M. (1993). La mujer de la ilusión. Buenos Aires: Paidós.

Fernández, A. M. (2001). Instituciones Estalladas. Buenos Aires: Eudeba.

Flaquer, LI. (1998). El destino de la familia. Barcelona: Ariel.

Foucault, M. (1980). Microfísica del poder. Madrid: Ediciones La Piqueta. 
Freixas, A. (2000). Entre el mandato y el deseo: el proceso de adquisición de la identidad sexual y de género. En C. Flecha y M. Núñez (Eds.), La Educación de las Mujeres: Nuevas perspectivas (pp. 23-32). Sevilla: Universidad de Sevilla.

Fridman, I. (2017). Mujeres y varones frente a las condiciones políticas del amor. Entre la autonomía y la soledad. En I. Meler. Psicoanálisis y género: escritos sobre el amor, el trabajo, la sexualidad y la violencia (pp. 165-175). Buenos Aires: Paidós.

Gimeno Collado, A (1999). La familia: el desafío de la diversidad. Barcelona: Ariel.

González Gabaldón, B. (1999). Los estereotipos como factor de socialización en el género. Comunicar 12, 79-88.

Gracia Fuster, E. \& Musitu Ochoa, G. (2000). Psicología social de la familia. Barcelona: Paidós.

Jodelet, D. (1986). La representación social: fenómenos, concepto y teoría. En S. Moscovici, (comp.), Psicología Social (pp. 469-494). Barcelona: Paidós.

Lagarde, M. (1998). Identidad genérica y feminismo. Sevilla: Instituto Andaluz de la Mujer. Laird, J.D. y Thomson, N.S. (1992). Psychology. Boston: Houghton Miffl in.

Martínez, A. (2011). Los cuerpos del sistema sexo/género: Aportes teóricos de Judith Butler. Revista de Psicología de la Facultad de Humanidades y Ciencias de la Educación de la Universidad Nacional de la Plata, (12), Año 2011-2012, 127-144. En Memoria Académica. Recuperado de http://www.memoria.fahce.unlp.edu.ar/art_revistas/pr.5641/pr.5641.pdf

Mead, G. H. (1963) La Persona (parte III) En Espíritu, persona y sociedad. Buenos Aires: Paidós.

Meler, I. (2017). Psicoanálisis y género: escritos sobre el amor, el trabajo, la sexualidad y la violencia. Buenos Aires: Paidós.

Morgade, G. (2012). Aprender a ser mujer. Aprender a ser varón. Relaciones de género y educación. Esbozo de un programa de acción. Buenos Aires- México: Novedades Educativas.

Moscovici, S. (1979) La representación social: un concepto perdido. En S. Moscovici. El Psicoanálisis, su imagen y su público (pp. 26-54). Buenos Aires: Huemul.

Mosterio García, J. (2010). Los estereotipos de género y su transmisión a través del proceso de socialización. En R. Radl Philipp Investigaciones Actuales de las Mujeres y el Género (pp. 239-251). Santiago de Compostela: Universidad Santiago de Compostela. 
Olavarría, J. (2012). ¿Nuevas masculinidades o masculinidades emergentes? Conferencia preparada para el Seminario de especialización Masculinidades y Género. Buenos Aires: Seminario PRIGEPP- FLACSO.

Ortega F. (1998). Imágenes y representaciones de género. Asparkía, 9, 9-20.

Ortiz Txabarri, I. (2015). La transmisión de los roles de género en los cuentos infantiles. Tesis de maestría. Universidad del País Vasco. http://hdl.handle.net/10810/15419

Ospina Botero, M., \& Montoya Pavas, E. (2015). Cambios en los estereotipos de género en la familia. Textos y Sentidos, 11, 141-154.

Pateman, C. (1995). El contrato sexual. Barcelona: Anthropos.

Quaresma Da Silva, D. y Ulloa Guerra, O. (2013). Estudios de masculinidades en la región oriental de Cuba. Athenea Digital, 13(2), 245-257. Recuperado de http://atheneadigital.net/article/viewFile/v13-n2-quaresmaulloa/1120-pdf-es

Quesada Jimenez, J. (2014). Estereotipos de Género y usos de la lengua. Un estudio descriptivo de las aulas y propuestas de intervención didáctica. Tesis doctoral. Universidad de Murcia.

Rosenberg, M. (2017) Comentario del artículo de Ana M. Fernández en Meler, I.

Psicoanálisis y género: escritos sobre el amor, el trabajo, la sexualidad y la violencia. Buenos Aires: Paidós.

Rubin, G. (1986). El tráfico de mujeres. Notas sobre una economía política del sexo. En Nueva Antropología, VIII (30). México: UNAM, 95-145.

Subirats, M. (1998). La educación de las mujeres: de la marginalidad a la coeducación. Propuestas para una metodología de cambio educativo. Serie Mujer y desarrollo, Santiago: Naciones Unidas.

Subirats, M. (2007). Ser hombre. En Castells, Manuel y Subirats, Marina, Mujeres y hombres ¿un amor imposible? (pp. 49-135). Madrid: Alianza Editorial. (Documentos PRIGEPP, 2012).

Wainerman, C. (Ed.) (2002). Familia, trabajo y género. Un mundo de nuevas relaciones. Buenos Aires: Fondo de Cultura Económica.

Williams, John E. and Deborah L. Best (1990), Measuring Sex Stereotypes, Newbury Park, CA: Sage Publications.

Williamson, D. S.; Bray, J. H. y Falicov, C. J. (1991). El desarrollo y cambios familiares a través de las generaciones: una perspectiva intergeneracional. En C. J. Falicov (Comp.), Transiciones de la familia: Continuidad y cambio en el ciclo de vida (pp. 491-527). Buenos Aires: Amorrortu. 


\section{Documento normativo:}

Código Civil y Comercial de la Nación. Ministerio Justicia y Derechos Humanos, Presidencia de la Nación. Recuperado de http://www.sde.gob.ar/justicia/cuadrocomparativo.pdf 